\title{
Contenidos de enseñanza de fracciones en 6to de primaria, México: Un análisis interconductual
}

\author{
Teaching contents of fractions in 6th grade, Mexico: An interbehavioral \\ analysis
}

\author{
César A. Hernández-Rodríguez ${ }^{1}$ \\ Comisión Nacional para la Mejora Continua en la Educación, México
}

\author{
Aldo Bazán-Ramírez ${ }^{2}$ \\ Consultor particular. Lima, Perú
}

Recibido: 25 - $05-20$

Aceptado: $12-06-20$

\section{Resumen}

El presente trabajo tuvo por objetivo analizar desde la taxonomía de Ribes y López (1985), los contenidos de la enseñanza de fracciones matemáticas en sexto grado de primaria. A partir de los aprendizajes esperados propuestos en los planes y programas para trabajar fracciones, además de realizar una revisión del libro de desafíos matemáticos 6to grado, se identificaron los ejercicios que los estudiantes tienen que realizar al trabajar con el tema de fracciones. Posterior a la revisión, se clasificaron los contenidos de acuerdo con la taxonomía funcional. Partiendo de la idea que las matemáticas se deberían trabajar desde niveles sustitutivos, al contar con su propio medio de contacto convencional, se pretendía encontrar actividades que promovieran dicho nivel de desligamiento funcional. Como principales resultados se encontraron: 1. Las actividades que promueven los libros no están de acuerdo con los aprendizajes esperados planteados en los planes y programas, 2. Las actividades en el libro de texto, así como los aprendizajes esperados resaltados en el plan y programa de estudios, corresponden a actividades de enseñanza y de aprendizaje de niveles más simples de desligamiento funcional de acuerdo con la taxonomía funcional asumida. Estas actividades en su mayoría de tipo procedimental, correspondería a un nivel de desligamiento de tipo suplementario.

Palabras clave: Nivel funcional; conductas sustitutivas; sexto de primaria; fracciones.

\footnotetext{
${ }^{1}$ Licenciado en psicología y Maestro en psicología, por la Universidad Autónoma del Estado de Morelos. Es especialista en evaluación educativa y actualmente trabaja en la Comisión Nacional para la Mejora Continua en la Educación, México. E-mail: cesalber.herod@gmail.com

ORCID: https://orcid.org/0000-0003-2121-0798

${ }^{2}$ Licenciado en psicología por la Universidad Nacional Federico Villarreal, Maestro en psicología y Doctor en psicología, ambos por la Universidad Nacional Autónoma de México. Es miembro del RENACYT de Perú; ha recibido la Medalla al mérito Alfonso Caso por la UNAM. Actualmente es consultor investigador en Evaluación, Educación y en Psicología Educativa, Perú. Autor para correspondencia. E-mail: abazanramirez@gmail.com ORCID: https://orcid.org/0000-0001-6260-5097

(C) Los autores. Este artículo es publicado por la Revista de Investigación en Psicología de la Facultad de Psicología, Universidad Nacional Mayor de San Marcos. Este es un artículo de acceso abierto, distribuido bajo los términos de la licencia Creative Commons Atribucion - No Comercia_Compartir Igual 4.0 Internacional. (http://creativecommons.org/licenses/by-nc-sa/4.0/) que permite el uso no comercial, distribución y reproducción en cualquier medio, siempre que la obra original sea debidamente citada.
} 


\begin{abstract}
The objective of this paper was to analyze, from the taxonomy of Ribes and López (1985), the contents of the teaching of mathematical fractions in basic education. Based on the expected learning proposed in the plans to work on fractions, in addition to doing a review of the book of mathematical challenges to know the exercises that students have to do when working with the topic of fractions. After the review, the contents were classified according to the functional taxonomy. Starting from the idea that mathematics should be worked from substitute levels, having its own conventional means of contact, it was intended to find activities that promote such level of functional detachment. As main results it was found that the activities that promote books are not according to the expected learning outlined in the plans and programs; in addition to the fact that the activities in the textbook, as well as the expected learning are stated in supplementary levels, therefore, they are mostly procedural.
\end{abstract}

Keywords: Funcional level; Sustitutive behavior; 6th grade; fractions.

Los planes y programas que sea analizaron para este trabajo corresponden al acuerdo 592, que entró en vigor en el 2011 y estuvo vigente hasta el 2016. No obstante, después de los cambios de gobierno y replantearse los planes y programas, solo los dos primeros grados de primaria conservaron los nuevos libros y planteamientos, pero los demás grados en Educación Básica, regresaron al plan 2011 y utilizar los libros de texto anteriores, por tal motivo, los recursos didácticos y libros de texto para sexto grado son los mismos, hasta que se planté la nueva reforma educativa. Uno de los principales retos para los nuevos planes es mejorar los desempeños de los estudiantes, principalmente en las asignaturas de español y matemáticas, por ser asignaturas elementales para el aprendizaje de otras asignaturas y el correcto desarrollo de otras disciplinas.

En lo que respecta a los planes y programas de estudio que plantea la SEP, en matemáticas, se trabaja con cuatro competencias que abarcan toda la educación primaria y secundaria, estas se denominan: Resolver Problemas de Manera Autónoma (RPMA), Comunicar Información Matemática (CIM), Manejar Técnicas Eficientemente (MTE) y Validar Procedimientos y Resultados (VPR). En sexto año de primaria, se divide el ciclo escolar en 5 bloques que se trabajan durante un año (SEP, 2011). En dicho plan se trabaja de manera integrada, de la tal manera que los contenidos estudiados en sexto año, sean complementarios a los temas revisados en años anteriores, así como necesarios para los temas que se trabajarán en secundaria (Bazán, Hernández, Castellanos \& Dávila, 2017).

Las competencias a pesar de tener una definición, no cuentan con criterios de logro definidos que permitan determinar si el estudiante que termina el sexto año de primaria cuenta o carezca de ellas, además de que tampoco existen criterios específicos al finalizar el tercero de secundaria. La falta de criterios de logro puede generar complicaciones en los profesores al determinar si un estudiante cuenta con las competencias planteadas en los planes y programas o inclusive hacer adecuaciones 
didácticas que permitan llevar al alumno a cumplir con ellas. Por lo que tampoco se sabe si será capaz de llevar estos conocimientos a su vida cotidiana.

A pesar de que los planes y programas no son el único factor que incide en la educación, sino que son varios los factores que interna o externamente afectan el logro académico alcanzado por los estudiantes (Bazán, Hernández, Hernández \& Ochoa, en prensa) es importante desde la psicología educativa favorecer y enriquecer la práctica educativa, de tal manera que se disminuyan los obstáculos en la educación (Guevara, 2006).

\section{Fracciones}

Se eligió el tema de las fracciones por ser un concepto matemático que se trabaja desde hace miles de años y que ha servido para la representación de cantidades que no son un entero. Sus primeros usos, fueron en la medición de distancias astronómicas, en donde los números enteros no eran tan precisos para hacer los cálculos necesarios para organizar la siembra o mejorar la observación de los astros. Hoy en día, las fracciones son un tema que se ve en los planes y programas de estudio de la primaria en diferentes grados, no obstante, se ha puesto en duda la practicidad de dedicarle temas a las fracciones cuando son representaciones que ya no tienen un uso predominante, debido a que en la mayoría de cálculos aritméticos se emplean los números decimales, en vez de las fracciones. Sin embargo, las fracciones son un tema importante para entender el concepto de número decimal.

Los babilonios hace casi 3000 años a. C. fueron los primeros en representar fracciones, las cuales, eran necesarias para sus observaciones astronómicas, ellos utilizaban una simbología de cuñas para hacer sus representaciones, por otro lado, los egipcios también hicieron uso de las fracciones para sus cálculos, sin embargo, su sistema de representación no era tan sencillo y complicaba mucho las operaciones aritméticas. En comparación con los babilonios, los avances egipcios no fueron muy grandes (Stewar, 2007).

El número racional se puede definir como una fracción $\mathrm{a} / \mathrm{b}$ donde a y $\mathrm{b}$ son números enteros ( $\mathrm{y}$ b es distinto de 0 ; de lo contrario, la fracción no tiene sentido). Las fracciones subdividen a los números enteros en partes arbitrariamente finas, de modo que, la longitud de una recta en una figura geométrica puede aproximarse tanto como queramos por un número racional.

\section{Modelos de representación}

Los modelos de representación dentro del aprendizaje de las fracciones son elementos importantes, debido a que forman parte del contacto que tendrán los estudiantes con las fracciones. Las maneras en que se pueden encontrar son distintas. Cada uno de estos modelos han sido estudiados y se conocen las dificultades que pueden tener en la manera de enseñarse. 
A. Modelo de área: En este modelo se trabaja a partir de figuras completas, las cuales pueden representar particiones en ellas o sombreados para representar fracciones. La figura representa la unidad, el número de partes en las que se divide representa el denominador, mientras que las partes sombreadas representan al numerador.

B. Modelo lineal:Se emplea una recta numérica para establecer las fracciones dentro de la línea. Esta puede representar un entero completo o inclusive más, dependerá de las marcas que contenga dentro de la línea.

C. Modelo de conjuntos: Este modelo implica una representación que emplea un grupo de elementos con o sin similitud de características dentro de un universo, de esta manera, se puede representar un entero o diferenciar el contenido dentro de la fracción a partir de señalar los objetos que conforman a la fracción. Este modelo implica el uso de cantidades discretas dentro de la representación y se puede ver su ejemplo en la figura

\section{Modelo Parte-todo}

Las críticas al modelo parte-todo o parte-parte, son en relación a que complejiza y dificulta el concepto de fracciones impropias, debido a que los ejercicios son enfocados en dividir un entero en diferentes partes, cuando la fracción se puede encontrar y trabajar en compañía de números enteros. Los ejercicios en el libro de texto incluyen las fracciones propias e impropias dentro de los ejercicios, pero desde el modelo trabajado, estos problemas pueden ser complicados para los estudiantes, sino tienen un referente que permita comprender de una mejor manera el trabajo con fracciones propias o impropias.

Este modelo implica ciertas limitantes para enseñar o provoca dificultades en los estudiantes, debido a que no nace de la matemática, sino que aparece como un recurso didáctico para enseñar el número fraccionario en el aula. Nace dentro de la práctica educativa (Escolano \& Gairín, 2007). Esta relación implica que la fracción representa siempre las partes de un todo unificado, lo cual representa la manera más arcaica de concebir a las fracciones, pero es el modelo más utilizado para enseñar este tema. No obstante, esto trae consigo algunas dificultades, como el error de comparar la fracción entre sus partes en vez de la comparación de la parte con un todo (Castro \& Torralbo, 2001). Es decir que el numerador y denominador se conciben como objetos separados y por lo tanto, la fracción pierde ante el estudiante la categoría de número, aparece como la relación simbólica de dos números naturales, como si fuera una simple entidad descriptiva.

Las relaciones que se establecen dentro del modelo parte-todo, son en su mayoría adquiridas de manera visual, lo que hace indispensable en la práctica didáctica, contar con representaciones de figuras geométricas regulares. Desde este modelo, se ignora la medida de magnitudes, por el hecho de trabajar la fracción 
como un sistema de repartición, olvidando sus orígenes como herramienta para realizar medidas más finas. De tal manera, los ejercicios o problemas planteados desde este modelo, se resuelven al hacer simples conteos, sin necesidad de medir (Escolano \& Gairín, 2007).

\section{La perspectiva interconductual}

Las matemáticas se utilizan para traducir al lenguaje matemático de los eventos, como ocurre en áreas como la física, sin embargo, sería un error sesgar a las matemáticas a simples símbolos, marcas o caracteres. En vez de limitarlas, las matemáticas se deben de trabajar o estudiar a partir de las relaciones que establece, como es el ejemplo del orden o la magnitud. Al final los resultados numéricos no se deben ver como objetos aislados, sino conectados a su traducción de origen (Kantor, 1933). Es decir, no ver los números como grafías en una hoja, sino entender que el número es la representación de una colección de objetos.

La psicología interconductal fue la teoría base, que se utilizó para llevar a cabo el análisis aquí presentado, que consiste en una revisión de los planes y programas de estudio, de cómo postulan la enseñanza del número racional o fraccionario y cómo se representan en los libros de texto.

La psicología interconductual, también tiene mucho que ofrecer a las matemáticas para favorecer su aprendizaje. Kantor (1967) nos dice que las matemáticas han tenido un problema de tipo conceptual, debido a que las matemáticas se han estudiado desde la concepción de una mente en donde suceden todos los procesos matemáticos, como un espacio separado del organismo y lejos de la interacción. Por ello, uno de las labores que tendría la psicología, sería hacer un análisis del estudio de las matemáticas como la interacción que tienen los individuos, entre este apoyo, estaría involucrado el aclarar la concepción matemática, la creación, intuición e inferencia y explicar las diversas formas de contacto con las relaciones como objetos de estímulo, para de esta manera, tener un mejor aprendizaje de las matemáticas.

Desde esta propuesta teórica, las matemáticas son como cualquier otra interconducta, empieza cuando el individuo comienza a abstraer relaciones de las situaciones ambientales. De ahí, vienen algunas interconductas como conteo, medición y otras técnicas culturales que se utilizan para llegar a resultados comunes que se difunden (Kantor, 1967).

Como se dijo anteriormente, el aprendizaje de las matemáticas son interconductas, de tal manera, son susceptibles a ser analizadas desde está postura teórica. Para tener una mejor claridad del tipo de interacción que se plantean en los planes y programas de estudio, el análisis se realizó utilizando las categorías propuestas desde la taxonomía de Ribes y López (1985) donde plantean que cualquier conducta puede ser categorizada en una escala que va de lo más sencillo, que sería una 
respuesta sujeta a lo que está presente en el momento con sus características físico químicas, como pueden ser actividades de atender o replicar. Hasta actividades más complejas, que sería una respuesta desligada de la situación concreta que implica acciones como teorizar o crear nuevos criterios.

\section{La Taxonomía funcional de la conducta}

La taxonomía planteada por Ribes y López (1985), se propone una categorización que clasificar cualquier tipo de interacción psicológica. Esta taxonomía consta de cinco niveles de complejidad, los cuales van desde interacciones sencillas, en donde la interacción sucede conforme a las propiedades físico químicas del ambiente y el sujeto no hace ningún cambio de la situación; hasta respuestas que implican situaciones más complejas, en donde es necesario desligarse de la situación para poder cumplir el criterio de logro establecido. Dentro de esta taxonomía el concepto de desligamiento funcional tiene una importancia fundamental. Es a partir de tres criterios de dónde se obtiene dicha clasificación: ajuste, mediación y desligamiento funcional. El ajuste es el requisito funcional que la interacción implica; la mediación por su parte, es el proceso por el que un estímulo o respuesta se vuelve crítico para dar estructura a la función (Tamayo \& González, 2018).

El desligamiento funcional es una característica esencial para definir la conducta psicológica, debido a que es lo que permite la diferenciación de una conducta biológica a una psicológica, la segunda implica tener una plasticidad interactiva con el ambiente (Ribes y López, 1958). Es decir que la conducta psicológica es modificable y variable a diferencia de la conducta biológica que es invariable (Kantor, 1933). El desligamiento funcional es la capacidad de separarse de las propiedades físico-químicas del ambiente o del estímulo.

Esta taxonomía busca delimitar de forma cualitativa el nivel de logro alcanzado en una interacción. Los niveles se clasifican en: Contextual, suplementario, selector, sustitutivo referencial y sustitutivo no referencial. El nivel alcanzado dependerá del desligamiento funcional que tenga el sujeto, es decir el desligamiento temporo-espacial que puede hacer el sujeto del estímulo. De esta manera la taxonomía le otorga validez y seguridad a las mediciones realizadas (Ribes \& López, 1985).Cada nivel de desligamiento funcional tiene características particulares:

a. El nivel contextual, es el más elemental, aquí el sujeto tiene una función muy pasiva, la cual podría ser de escucha o repetición, no requiere un desligamiento de la situación;

b. El nivel suplementario requiere de un desligamiento del momento y el espacio más elaborado, ya que en este nivel el sujeto influye en el ambiente, realiza actividades como seguir un procedimiento, previamente enseñado; 
c. En el nivel selector el alumno elige un procedimiento a seguir o da una respuesta, dependiendo de un criterio dado por un profesor, por ejemplo, elegir el resultado correcto en una pregunta que tenga cuatro opciones (Ribes y López, 1985; Guevara, 2006; Varela, 2008).

Estos tres primeros niveles de la taxonomía, también se pueden mencionar como niveles Intrasituasionales, puesto que suceden dentro de la situación planteada. A partir del cuarto nivel, es necesario que el individuo cuente con sistema convencional de interacción, esto quiere decir que tenga los elementos para poder interactuar, un ejemplo clásico de un sistema reactivo convencional es el lenguaje, no obstante, no es el único.

d. Nivel sustitutivo referencial requiere de un desligamiento funcional más elaborado, ya que el alumno tiene que referir aspectos que no se encuentran en ese momento, en este nivel, el alumno puede narrar hechos o describir personajes; $y$

e. El nivel sustitutivo no referencial, es el más alto en esta taxonomía e implica un desligamiento absoluto de los eventos concretos, puede referir aspectos que no están presentes y además agregar ideas propias o juicios de opinión, donde se incluyen razonamientos científicos o morales.(Ribes y López, 1985; Guevara, 2006; Varela, 2008).

Los últimos dos niveles de desligamiento funcional, implican también situaciones distintas, en el caso de sustitutivo referencial, se conoce como una conducta Extrasituacional, ya que uno está en contacto físico con el aquí y el ahora, son que puede hacer referencia de aspectos ajenos a la situación, como por ejemplo recordar lo que dijo una persona en otro lugar. El nivel sustitutivo no referencial, se asocia con interconductas de tipo Transituacional, ya que suceden fuera de las características temporo-espaciales, el organismo asocia dos o más respuestas convencionales conocida como conductas transituacionales (Tamayo \& González, 2018).

Por todo lo anterior, el objetivo fue realizar un análisis desde la taxonomía de Ribes y López (1985) de los contenidos de la enseñanza de las fracciones matemáticas en educación básica, empleando los planes, programas y libros de texto como insumos para dicho análisis.

\section{MÉTODO}

\section{Diseño}

En este sentido, el diseño en este trabajo fue cualitativo y de análisis teórico al comparar los planes y programas de estudio en el tema de fracciones y el análisis de conducta. Para ello, se realizó una descripción de los planes y programas 
del programa que comprende el acuerdo 592 (SEP, 2011).Para poder hacer el análisis, se tomaron como categorías la taxonomía de Ribes y López (1985), con la intención de conocer el tipo de interacción que se proponen en los planes y libros de texto.

\section{Procedimiento}

Con la idea de contar con una radiografía completa de la enseñanza de las fracciones, no sólo se realizó el análisis de sexto grado (grado donde se realizará la planeación), sino desde el grado donde comienza la enseñanza de las fracciones, que comienza en el tercer grado.

Los planes y programas fueron tomados del acuerdo 592 propuesto en el 2011. Para un mejor análisis, se revisó el currículo y aprendizajes esperados como se muestra en la figura 1.

Como se puede observar en la figura 1, se presentan las competencias matemáticas que se favorecen (Son las mismas para todos los grados), los aprendizajes esperados para para el bloque y los tres ejes. Cada eje con los temas que pretende revisarse. El análisis para este trabajo, se concentró principalmente en los aprendizajes esperados.

\begin{tabular}{|c|c|c|c|}
\hline APRENDIZAJES ESPERADOS & \multicolumn{3}{|c|}{ EJeS } \\
\hline $\begin{array}{l}\text { - Resuelve problemas que } \\
\text { impliquen leer, escribir y } \\
\text { comparar números naturales, } \\
\text { fraccionarios y decimales, } \\
\text { explicitando los criterios de } \\
\text { comparación. } \\
\text { - Resuelve problemas aditivos } \\
\text { con números naturales, } \\
\text { decimales y fraccionarios } \\
\text { que implican dos o más } \\
\text { transformaciones. } \\
\text { - Describe rutas y calcula la } \\
\text { distancia real de un punto } \\
\text { a otro en mapas. }\end{array}$ & $\begin{array}{l}\text { Nümeros y SISTEMAS } \\
\text { DE Numeración } \\
\text { - Lectura, escritura y } \\
\text { comparación de números } \\
\text { naturales, fraocionarios } \\
\text { y decimales. Explicitación } \\
\text { de los criterios de comparación. } \\
\text { Prosuevas adrtivos } \\
\text { - Resolución de problemas } \\
\text { aditivos con números } \\
\text { naturales, decimales y } \\
\text { fraccionarios, variando la } \\
\text { estructura de los problemas. } \\
\text { Estudio o reafirmación de los } \\
\text { algoritmos convencionales. } \\
\text { Problemas mutripucativos } \\
\text { - Resolución de problemas } \\
\text { multiplicativos con valores } \\
\text { fraccionarios o decimales } \\
\text { mediante procedimientos } \\
\text { no formales. }\end{array}$ & $\begin{array}{l}\text { Figuras y cuerpos } \\
\text { - Identificación de los ejes de } \\
\text { simetría de una figura (poligonal } \\
\text { o no) y figuras simétricas } \\
\text { entre sí, mediante diferentes } \\
\text { recursos. } \\
\text { Usicación Espacial } \\
\text { - Elección de un código para } \\
\text { comunicar la ubicación de } \\
\text { objetos en una cuadrícula. } \\
\text { Establecimiento de códigos } \\
\text { comunes para ubicar objetos. } \\
\text { Medioa } \\
\text { - Cálculo de distancias reales } \\
\text { a través de la medición } \\
\text { aproximada de un punto a otro } \\
\text { en un mapa. }\end{array}$ & 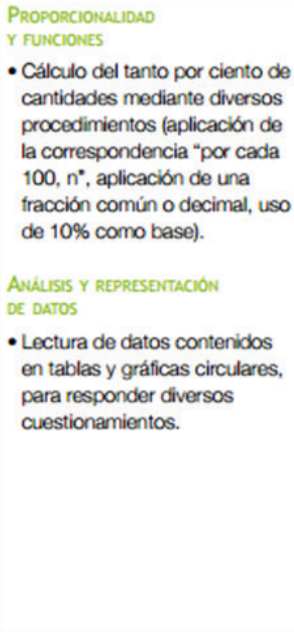 \\
\hline
\end{tabular}

Figural.Mapa curricular del bloque 1 de matemáticas sexto grado (SEP, 2011). 
Además de la planeación, era importante realizar un análisis de los ejercicios en los que los alumnos tenían contacto en el salón de clases. Por ello, se revisaron los libros de texto, denominados desafíos matemáticos de tercero a sexto grado de primaria. Revisando los modelos que se utilizan para enseñar, además del número de lecciones y complejidad de los ejercicios que tienen que resolver los estudiantes. Parte del análisis consistió también en comparar si los ejercicios propuestas en los libros tenían lógica con lo estipulado en los aprendizajes esperados del currículo.

Una vez revisados los aprendizajes esperados en el currículo y los ejercicios en los libros de texto, se clasificaron a partir del desligamiento funcional que promueven en los estudiantes. Este análisis tiene su fundamento debido a que las matemáticas por tener su propio medio de contacto convencional, las actividades que se deberían de promover serían sustitutivas, de tal manera que se buscó si las actividades propuestas promovían este nivel de desligamiento funcional o en su defecto, conocer los niveles en los que se trabaja en las aulas mexicanas.

\section{RESULTADOS}

Dentro de los planes y programas de estudio de la SEP, se trabaja desde tres ejes temáticos las matemáticas que son: Sentido Numérico y Pensamiento Algebráico, Forma Espacio y Medida y Manejo de la Información. Las fracciones a pesar de ser una herramienta para trabajar la medida, el contenido de las fracciones se concentra en el eje de Sentido Numérico y Pensamiento Algebraico (SEP 2011). Esto debido a que se trabaja la fracción como herramienta para la repartición de figuras o para hacer comparaciones de equivalencias con números decimales principalmente, aunque también se encuentra la comparación con los porcentajes, pero en un menor grado. Para una mejor revisión, se presentan el análisis realizado por cada uno de los grados.

El análisis de los planes y programas muestran que desde el tercer grado de primaria las fracciones comienzan a ser trabajadas en los salones de clases. Acabando el tercer grado de primaria, de acuerdo con el programa, en el eje temático de sentido numérico y pensamiento algebraico, los estudiantes deberían poder operar adiciones utilizando medios, cuartos y octavos. Son cuatro los aprendizajes esperados planteados en los programas, pero estos no coinciden con las cuatro lecciones presentadas en los libros, donde, además, sólo hay un ejercicio que involucra suma, en la tabla 1 se puede apreciar la distribución de las lecciones en los diferentes bloques, cabe destacar que las actividades presentadas en las cuatro lecciones son de tipo suplementarias.

Para cuarto grado de primaria, se trabaja fundamentalmente las equivalencias de fracciones y la suma y resta de fracciones para resolver problemas matemáticos 
sencillos, de 18 lecciones que hay en el libro de desafíos matemáticos para cuarto grado, sólo hay una lección que implica la operación de fracciones, las 17 restantes trabajan principalmente las fracciones desde su equivalencia o utilizándola como operador para repartir un entero entre un cierto número de personas o cosas (SEP, 2014a; SEP, 2014b; SEP, 2011).

\section{Tabla 1}

Características del libro de Tercer grado

\begin{tabular}{ll}
\hline \multicolumn{1}{c}{ Aspecto } & \multicolumn{1}{c}{ Descripción } \\
\hline Número de lecciones que presentan & 4 lecciones: 1 en Bloque III, 1 enBloque IV y 2 en Bloque V) \\
Modelo de representación & Modelo de área y representaciones continuas o escritas. \\
Modelos de enseñanza & Modelo parte-todo \\
Nivel funcional & Suplementario \\
\hline
\end{tabular}

En la tabla 2 se puede observar a detalle la descripción del libro de cuarto grado, cabe mencionar que de acuerdo al plan de estudios, sólo hay seis aprendizajes esperados, es decir que practicamente hay el triple de lecciones por las actividades en el libro. Al igual que en tercer grado, las actividades en los libros y planes están en un nivel de desligamiento funcional suplementario, por ser actividades procedimentales.

\section{Tabla 2}

Características del libro de Cuarto grado

\begin{tabular}{ll}
\hline \multicolumn{1}{c}{ Aspecto } & \multicolumn{1}{c}{ Descripción } \\
\hline Número de lecciones que presentan & $\begin{array}{l}\text { 18 lecciones: 4 en bloque I, 3 lecciones en bloque II, 4 en bloque III, 4 del } \\
\text { bloque IV y 3 del bloque. }\end{array}$ \\
Modelo de representación & Modelo parte-todo \\
Modelos de enseñanza & Modelo lineal, de área y de conjunto \\
Nivel funcional & Suplementario \\
\hline
\end{tabular}

En el quinto grado, el número de lecciones es menor a la de cuarto, con sólo 16 lecciones, de las cuales tres se dedican a la operación, mientras que las demás están enfocadas en las equivalencias y comparaciones de fracciones, en la tabla 3 , se pueden apreciar las características del libro de quinto grado. La mayoría de las actividades presentadas en este grado tienen que ver con la operación de adisión y sustracción de fracciones, las cuales esta en coherencia con los 7 aprendizajes esperados encontrados en el plan de estudios, que esta enfocado a las sumas y restas. No obstante el nivel de desligamiento funcional planteado para estás actividades son sulpementarias en su mayoría, con algunas actividades del nivel selector. 
Tabla 3

Características del libro de Quito grado

\begin{tabular}{ll}
\hline Aspecto & Descripción \\
\hline Número de lecciones que presentan & $\begin{array}{l}\text { 16 Lecciones: 4 bloque I, 3 del bloque II, 4del bloque III, 4 del bloque IV y } \\
1 \text { de bloque V. }\end{array}$ \\
Modelo de representación & Modelo parte-todo \\
Modelos de enseñanza & Modelo de conjunto y lineal \\
Nivel funcional & Suplementario y Selector \\
\hline
\end{tabular}

Por último en sexto grado, lo que se pretende enseñar aparte de seguir con las lecciones de equivalencia y repartición, es el que los estudiantes aprendan a multiplicar y dividir utilizando las fracciones. Dentro del plan de estudios, se menciona la posibilidad de trabajar la multiplicación y división de la fracción con un número natural, sin embargo, dentro de los ejercicios del libro de desafíos matemáticos se encuentra una sola lección que implica la multiplicación de una fracción con un número natural, pero en ninguna lección viene la posibilidad de dividir la fracción con número natural o viceversa, en la tabla 4 , se ve claramente cómo está representado(SEP, 2014c; SEP, 2014d; SEP, 2011).

\section{Tabla 4}

Características del libro de Sexto grado

\begin{tabular}{ll}
\hline \multicolumn{1}{c}{ Aspecto } & \multicolumn{1}{c}{ Descripción } \\
\hline Número de lecciones que presentan & $\begin{array}{l}\text { 21 Lecciones: 4 bloque I, 3 del bloque II, } 4 \text { de bloque III, 7 del } \\
\text { bloque IV y 3 del bloque V. }\end{array}$ \\
Modelo de representación & Modelo parte-todo \\
Modelos de enseñanza & Modelo lineal \\
Nivel funcional & Suplementario \\
\hline
\end{tabular}

A continuación en la tabla 5, se pueden observar los aprendizajes esperados encontrados en los planes de estudio, en dónde se puede observar que predominan los aprendizajes a un nivel suplementario, en otras palabras, solo se está replicando un algoritmo para seguir unprocedimiento. Por otro lado, sólo se encontró dos actividades a nivel sustitutiva referencial y ninguna a un nivel sustitutiva no referencial.

Por otro lado, detro de los libros de texto se puede apreciar el uso de modelos para la enseñanza de fracciones, como es el modelo lineal, de conjunto y de área, su uso es diferente conforme el grado. Por ejemplo, en tercero y cuarto grado predomina el modelo de área para el trabajo con las fracciones, mientras que en quinto y sexto, es mayoritariamente el modelo lineal, para trabajar las fracciones dentro de la recta numérica. 


\section{Tabla 5}

Nivel de desligamiento funcional en el plan de estudios

\begin{tabular}{|c|c|c|}
\hline Bloque & Aprendizaje Esperado & $\begin{array}{l}\text { Nivel de aptitud } \\
\text { funcional }\end{array}$ \\
\hline I & $\begin{array}{l}\text { Lectura, escritura y comparación de números naturales, fraccionarios y deci- } \\
\text { males. Explicitación de los criterios de comparación. }\end{array}$ & Contextual \\
\hline I & $\begin{array}{l}\text { Resolución de problemas aditivos con números naturales, decimales y fraccio- } \\
\text { narios, variando la estructura de los problemas. Estudio o reafirmación de los } \\
\text { algoritmos convencionales. }\end{array}$ & Suplementaria \\
\hline I & $\begin{array}{l}\text { Resolución de problemas multiplicativos con valores fraccionarios o decimales } \\
\text { mediante procedimientos no formales. }\end{array}$ & Selectoras \\
\hline II & $\begin{array}{l}\text { Ubicación de fracciones y decimales en la recta numérica en situaciones } \\
\text { diversas. Por ejemplo, se quieren representar medios y la unidad está dividida en } \\
\text { sextos, la unidad no está establecida, etcétera. }\end{array}$ & Suplementaria \\
\hline III & $\begin{array}{l}\text { Identificación de una fracción o un decimal entre dos fracciones o decimales } \\
\text { dados. Acercamiento a la propiedad de densidad de los racionales en contraste } \\
\text { con los números naturales. }\end{array}$ & $\begin{array}{l}\text { Sustitutiva } \\
\text { referencial }\end{array}$ \\
\hline IV & $\begin{array}{l}\text { Conversión de fracciones decimales a escritura decimal y viceversa. Aproxi- } \\
\text { mación de algunas fracciones no decimales usando la notación decimal. }\end{array}$ & Selectoras \\
\hline IV & $\begin{array}{l}\text { Identificación y aplicación de la regularidad de sucesiones con números (natu- } \\
\text { rales, fraccionarios o decimales) que tengan progresión aritmética o geométrica, } \\
\text { así como sucesiones especiales. Construcción de sucesiones a partir de la } \\
\text { regularidad. }\end{array}$ & Suplementaria \\
\hline IV & $\begin{array}{l}\text { Resolución de problemas que impliquen calcular una fracción de un número } \\
\text { natural usando la expresión "a/b de } n "\end{array}$ & $\begin{array}{l}\text { Sustitutiva } \\
\text { referencial }\end{array}$ \\
\hline V & $\begin{array}{l}\text { Resolución de problemas que implique una división de numero fraccionario o } \\
\text { decimal entre un número natural. }\end{array}$ & Suplementaria \\
\hline
\end{tabular}

Nota: Los aprendizajes esperados presentados en esta tabla, fueron tomadas del acuerdo 592 (SEP, 2011).

Los ejericios establecidos en los libros de desafios matemáticos no cuentan con una complejidad gradual, sino que se repite el mismo ejercicio cambiando la fracción a representar o las cifras en la suma, pero el alumno no tiene que desarrollar nada nuevo, un ejemplo es el que se puede apreciar en la figura 10, en dónde el mismo ejercicio no cambia su complejidad, la figura representa un fragmento de la lección, pero como en el ejemplo todavía hay 6 rectas más en el que sólo cambias las cifras.

Formen parejas y ubiquen en las rectas numéricas los números que se indican.

a) 1

b) 2.5

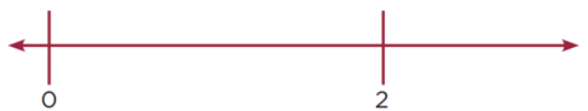

c) 1

d) $\frac{1}{2}$

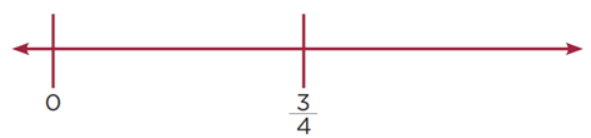

Figura2. Ejemplo tomado del libro de sexto año de Desafíos Matemáticos (SEP, 2011). 
Dentro de los ejercicios propuestos para los libros de texto en matemáticas se trabaja con los tres modelos, aunque de una manera desequilibrada, dándoles mayor énfasis al modelo de área y al modelo lineal. Aunque también se encuentra el modelo de conjunto, pero en un grado menor, en comparación de los otros dos.

Por último, cabe señalar que el modelo parte-todo o parte-parte que se encuentra de alguna manera implícito en los libros de texto, involucra ciertas dificultades que se pueden encontrar en los cuatro grados analizados. El constante uso de la fracción en ejercicios de equivalencia puede contribuir a entender el número fraccional como una simple situación descriptiva. El plan de estudios, tiene una incongruencia en la relación parte-todo, debido a que no se trabaja el significado de cociente y de razón, en el caso de la fracción como operador, se localiza un ejercicio donde se emplea.por lo que quizá tampoco sea suficiente para comprender o desarrollar en los estudiantes el aprendizaje de los diferentes usos que se le da al número fraccionario.De esta manera, la fracción queda como un simple descriptor, en el mejor de los casos, de medida, aunque dentro del programa para el profesor, en los objetivos de aprendizaje se encuentra como un punto a trabajar la fracción desde sus cuatro conceptos: Operador, Razón, Medida y Cociente (SEP, 2011b).

\section{DISCUSIÓN}

El objetivo planteado en este trabajo, fue analizar con base en una taxonomía de organización funcional de la conducta (Ribes \& López, 1985), los contenidos para la enseñanza de las fracciones matemáticas en la educación básica en México. De acuerdo con los resultados obtenidos, a lo largo de los temas y lecciones en los libros de texto y los programas de matemáticas del tercer al sexto grados, no se aprecia un modelo de enseñanza de las fracciones en general. Pareciera que son varios temas o conceptos que se pueden trabajar con las fracciones, pero sin seguir un modelo específico.

Es preocupante esta poca atención a la enseñanza de las fracciones en niveles complejos de comprensión y aplicación. La fracción es un tema importante para el desarrollo de diferentes conceptos matemáticos, desde su nacimiento, se originaron por una necesidad de medidas más precisas. Actualmente se les ha restado importancia por la facilidad de operación al usar número decimal, no obstante, el número decimal, no es tan exacto como la fracción.

Un aspecto a resaltar es que, las actividades propuestas en los aprendizajes esperados en sexto grado, corresponden en su mayoría a un nivel suplementario de desarrollo y desligamiento funcional del comportamiento, y están enfocados a cumplir un algoritmo para dar una respuesta. Asimismo, en los ejercicios del libro de sexto grado de primaria, las actividades están planteadas también en un nivel suplementario. El alumno solo debe seguir un procedimiento para poder cumplir 
con el criterio de logro, aunado a ello, las preguntas o problemas no cambian de complejidad en una misma lección, sólo cambian las variables que se presentan.

El trabajar sólo con actividades de tipo suplementario, no permite al estudiante realizar una correcta transferencia de conocimiento o de sus habilidades al momento de responder tareas que implican otro nivel más complejo de desligamiento funcional. De tal manera que los estudiantes responderán en un nivel suplementario en los exámenes o actividades que le proporcionen los profesores (Hernández, 2016). Pero al solicitar que el estudiante ejecute demandas de logro de acuerdo con niveles sustitutivos, este no será competente.

Un punto importante a resaltar es la incongruencia que se presenta entre los niveles funcionales que enuncian los aprendizajes esperados en el programa de estudios y el tipo de actividades planteadas en los libros de texto, debido a que en el caso de que el docente quiera llegar a los niveles planteados, tendrá que ser a partir de actividades diseñadas por el mismo. Por ejemplo, en la tabla 5, se puede observar que en el bloque I hay tres actividades, las cuales van aumentando desde el nivel contextual hasta el selector, no obstante, las actividades que hay en el bloque I de actividades en el libro, son sólo actividades suplementarias. Por otro lado, el caso más complicado es en el bloque IV, debido a que presenta un aprendizaje esperado suplementario y uno sustitutivo referencial, no obstante, nos encontramos nuevamente siete actividades en el mismo bloque con nivel suplementario.

Asimismo, las diferencias en lo que implica las operaciones, en los planes y programas, pareciera que la operación con fracciones es la manera más eficaz de medir el conocimiento de las fracciones: en tercero sumas de medios cuartos y octavos, en cuarto grado sumas y restas con problemas sencillos, para quinto se continua trabajando con sumas y restas de fracciones, mientras que en sexto grado se requiera multiplicar y dividir fracciones. La complejidad de las operaciones se complican, pero el número de lecciones y por lo tanto de ensayos de estas operaciones tienden a ser muy escasas, limitándose en algunos grados en una sola lección en todo el ciclo escolar, sin mencionar que se dedican tres grados para la adición y sustracción y sólo el último grado para la multiplicación y división, que si bien, el algoritmo para resolverlo es sencillo, la explicación del uso u origen de ese algoritmo no se enseña.

El tipo de interacción propuestas para los estudiantes en el Plan y Programas para la primaria, concuerda con lo encontrado por Olfos (2001), respecto a que la práctica docente en matemáticas se limita a actividades concretas, disminuyendo o eliminando las actividades abstractas para los estudiantes. Parte de las conclusiones con base en sus observaciones, es que la práctica docente ha llevado al profesor a preferir actividades sencillas y procedimentales e incluso saltarse actividades que podrían provocar preguntas por parte de los estudiantes al profesor.Asimismo, estos resultados confirmaron que el contenido matemático que se 
enseña, es a partir de fuentes de significado limitados, al sólo tomar el dominio numérico, obviando ideas importantes que podrían conectarse con otros dominios matemáticos, como geometría o algebra (Butto, Delgado, \& Bazán, 2018).

De la misma manera, el uso del modelo parte-todo o parte-parte, como una respuesta de la práctica docente para enseñar fracciones (Escolano \& Gairín, 2007), promueve ejercicios con procedimientos sencillos, es decir, al alumno sólo se le exige hacer conteos en las representaciones, nuevamente actividades de tipo suplementarias. El problema se encuentra al momento de operar con las fracciones, hecho evidenciado en el desempeño de los estudiantes en los resultados en la prueba EXCALE exclusivamente en las preguntas que implicaban operar con fracciones (EXCALE, 2012).

Uno de estos problemas es que los objetivos planteados al final de la planeación, son aspectos que no se pueden observar, por lo tanto, es difícil para el profesor, saber si se ha cumplido el objetivo de la planeación. Otro problema es que carece de nivel de destreza, es decir, que no hay una especificación de la dificultad que puede tener llegar al objetivo, por lo que tampoco permite que haya una secuencia para ir desde lo más sencillo a lo más complejo (Guevara, 2006). Existe una carencia de explicitación de criterios que deben cumplir de los estudiantes, para el aprendizaje de las matemáticas (Ribes \& Varela, 1994) de formaa cí́tica, reflexiva y que tenga aplicación en contextos del mundo real, a nivel perosnal, colectivo, ocupacional y científico (OECD, 2013), y puedan tener una inserción real y concreta a la sociedad.

Es importante para que haya un cambio en las respuestas de los estudiantes que las actividades o situaciones de enseñanza aprendizaje estén elaboradas en niveles de desligamiento funcional sustitutivos, referencial y no referencial, para que los estudiantes puedan trabajar las matemáticas como la traducción al lenguaje matemático de los eventos, lo cual permitirá a los alumnos trabajar las matemáticas desde sus teoremas y axiomas sin limitarse a un simple conteo de caracteres o representación simple.

Estos resultados pueden dar un punto de partida para la creación de tecnologías desde dos vías, por un lado, se podría emplear el mismo sistema de análisis para revisar diferentes contenidos en la asignatura de matemáticas, con la finalidad de analizar el nivel funcional con el que los estudiantes están trabajando. Por otro lado, la enseñanza de fracciones desde los modelos parte todo o parte parte, nos pueden dar luz para diseñar una planeación didáctica que integre los elementos de la teoría interconductual, como se ha propuesto en otros trabajos (Ribes, 2008). De esta manera se buscaría que las matemáticas sean trabajadas desde situaciones extrasituacionales y transituacionales, proporcionado a los estudiantes mejores competencias. 


\section{CONCLUSIONES}

De acuerdo con lo revisado en el trabajo se puede decir que el objetivo del trabajo se cumplió al analizar los diferentes recursos didácticos con los que son enseñadas las fracciones en sexto de primaria, de tal manera, que queda claro que los niveles o criterios en los diferentes grados son de niveles funcionales suplementarios, lo que implican un nivel muy concreto. Es importante que los nuevos planes y programas, así como los libros nuevos, contengan contenido de niveles sustitutivo referencial y sustitutivo no referencial, que son, desde donde se deberían de trabajar los contenidos matemáticos.

También es importante generar materiales que cuenten con la congruencia entre los planes y programas y los libros de texto a los que tienen acceso los estudiantes, debido a que esté análisis evidenció que los requerimientos u objetivos a cumplir en los planes y programas, son diferentes a las actividades presentadas en los libros de texto, lo cual podría crear una desventaja al momento de realizar evaluaciones que pretendan evaluar lo que dicen los planes y programas.

El modelo interconductual, permitió un análisis estructurado de los contenidos matemáticos en los planes, programas y libros de texto, por lo cual la metodología de análisis empleada, sirvió para alcanzar satisfactoriamente los resultados del trabajo.

Los resultados obtenidos, permitirán dejar un antecedente para replantear los contenidos de las fracciones en los nuevos planes, programas y libros de texto o por lo menos, evidenciar que el nivel funcional con el que se trabajan el tema de las fracciones en matemáticas es muy sencillo. Sería importante, realizar este análisis para otros contenidos matemáticos con la finalidad de conocer con qué nivel funcional de trabaja en la primaria.

\section{REFERENCIAS}

Bazán, A., Hernández, C., Castellanos, D., y Dávila, M. (2017). Logro en ENLACE, competencias, calificaciones y contexto de procedencia al terminar la primaria. En M.L. Madueño, L. Serna, \& A. Manig (Coordinadores), Investigaciones educativas Una mirada hacia los actores, los procesos, y las prácticas de formación, 11-27. México: Tabook.

Bazán-Ramírez, A., Hernández-Padilla, E., Hernández, C. \& Ochoa, E. (En prensa). Relaciones entre contexto de procedencia y logro académico al terminar la primaria. Revista Electrónica de Investigación Educativa, 22, e08, 1-16. https://doi.org/10.24320/ redie.2020.22.e08.2335

Butto-Zarzar, M. C., Delgado-Fernández, J., \& Bazán-Ramírez, A. (2018). Procesos de generalización: Una vía de acceso al pensamiento algebraico temprano en educación básica. Horizontes Pedagógicos issn-1:0123-8264, 21 (2), 25 -36.Obtenido de: https:// revistas.iberoamericana.edu.co/index.php/rhpedagogicos/article/view/1269 
Castro, E., \& Torralbo, M. (2001). Capítulo 12 Fracciones en el currículo de la educación primaria. En M. E. C., Didáctica de la matemática en la educación primaria (págs. 285-314). España : Síntesis.

Escolano, R., \& Gairín, J. M. (2007). Enseñanza del número racional positivo en educación primaria: propuesta didáctica con modelos de medida. Investigaciones en Educación Matemática: pensamiento numérico, 185-212.

Excale. (2016). Resultados de Excale Matemáticas 2013-2014. México: INEE.

Guevara, B. C. (2006). Análisis interconductual de algunos elementos que constituyen la enseñanza básica. Revista Mexicana de Investigación Educativa, 1037-1064.

Hernández, C. (2016). Planeación desde un enfoque interconductual para generar competencias matemáticas en primaria. (Tesis Inedita de Maestría): Universidad Autónoma del Estado de Morelos, México.

Kantor, J. R. (1933). A survey of the science of psychology. Bloomington: Principia press.

Kantor, J. R. (1967). Psicología Interconductual un ejemplo de construcción científica sitemática . E.U. : The principia press, 2da edición.

Kantor, J. R. (1977). Psychological linguistics. Chicago: Principia press.

OECD (2013). PISA 2012. Assessment and Analytical Framework: Mathematics, Reading, Science, Problem Solving and Financial Literacy. OECD Publishing. http:// dx.doi.org/10.1787/9789264190511-en.

Olfos, R. (2001). Entendiendo la clase de matemáticas. Revista latinoaméricana de investigación matemática educativa, 23-43.

Ribes, E. I., \& López, F. (1985). Teoría de la conducta. Un análisis de campo y paramétrico. México: Trillas.

Ribes, E. I., \& Varela, J. B. (1994). Evaluación interactiva del comportamiento inteligente: Desarrollo de una metodología computacional. Revista Mexicana de la Conducta, 83-97.

Ribes, E. I. (2008) Educación básica, desarrollo psicológico y planeación de competencias. Revista mexicana de psicología, 193-207

Secretaria de Educación Pública. (2011). Plan de estudios de la SEP 2011. México: SEP.

Secretaría de Educación Pública. (2014). Desafios Matemáticos Cuarto Grado . México: Secretaría de Educación Pública.

Secretaría de Educación Pública. (2014). Desafios Matemáticos Quinto grado . México: Secretaría de Educación Pública.

Secretaria de Educación Pública. (2014). Desafios matemáticos sexto grado. México : Secretaria de Educación Pública .

Secretaría de Educación Pública. (2014). Desafios Matemátios Tercer grado . México : Secretaría de Educación Pública. 
Stewar, I. (2007). Historia de las matemáticas en los últimos 10,000 años. Barcelona: Critica.

Tamayo, J. E. \& González, V. H. (2018). Evaluación de la facilitación interfuncional en las funciones intrasituacionales. Psicología y Educación 12, 18-35.

Varela, J. (2008). Conceptos básicos del Interconductismo. Guadalajara, Jalisco: Universidad de Guadalajara. 